\title{
Pressure Effects in Supercooled Water: Comparison between a 2D Model of Water and Experiments for Surface Water on a Protein.
}

\author{
Giancarlo Franzese ${ }^{1}$, Kevin Stokely ${ }^{2}$, Xiang-qiang $\mathrm{Chu}^{3}$, \\ Pradeep Kumar ${ }^{2,4}$, Marco G. Mazza ${ }^{2}$, Sow-Hsin Chen ${ }^{3}$, and \\ H. Eugene Stanley ${ }^{2}$ \\ ${ }^{1}$ Departament de Física Fonamental, Universitat de Barcelona, Diagonal 647, 08028 \\ Barcelona, Spain \\ ${ }^{2}$ Center for Polymer Studies and Department of Physics, Boston University, Boston, \\ MA 02215 USA \\ ${ }^{3}$ Department of Nuclear Science and Engineering, Massachussetts Institute of \\ Technology, Cambridge, MA 02139 USA \\ ${ }^{4}$ Center for Studies in Physics and Biology, Rockefeller University, New York, NY \\ 10021 USA \\ E-mail: gfranzese@ub.edu
}

\begin{abstract}
.
Water's behavior differs from that of normal fluids, having more than sixty anomalies. Simulations and theories propose that many of these anomalies result from the coexistence of two liquid phases with different densities. Experiments in bulk water confirm the existence of two local arrangements of water molecules with different densities, but, because of inevitable freezing at low temperature $T$, cannot ascertain whether the two arrangements separate into two phases. To avoid the freezing, new experiments measure the dynamics of water at low $T$ on the surface of proteins, finding a crossover from a non-Arrhenius regime at high $T$ to a regime that is approximately Arrhenius at low T. Motivated by these experiments, Kumar et al. [Phys. Rev. Lett. 100, 105701 (2008)] investigated, by Monte Carlo simulations and mean field calculations on a cell model for water in two dimensions (2D), the relation of the dynamic crossover with the coexistence of two liquid phases. They show that the crossover in the orientational correlation time $\tau$ is a consequence of the rearrangement of the hydrogen bonds at low $T$, and predict that:

(i) the dynamic crossover is isochronic, i.e. the value of the crossover time $\tau_{\mathrm{L}}$ is approximately independent of pressure $P$;

(ii) the Arrhenius activation energy $E_{\mathrm{A}}(P)$ of the low- $T$ regime decreases upon increasing $P$;

(iii)the temperature $T^{*}(P)$ at which $\tau$ reaches a fixed macroscopic time $\tau^{*} \geq \tau_{\mathrm{L}}$ decreases upon increasing $P$; in particular, this is true also for the crossover temperature $T_{\mathrm{L}}(P)$ at which $\tau=\tau_{\mathrm{L}}$.

Here, we compare these predictions with recent quasielastic neutron scattering (QENS) experiments performed by X.-Q. Chu et al. on hydrated proteins at different values of $P$. We find that the experiments are consistent with these three predictions.
\end{abstract}


Pressure Effects in Supercooled Water: Comparison between a 2D Model of Water and Experiments for Surf

\section{Introduction}

Water has many anomalies compared to normal liquids [1. Experiments show that its thermodynamics fluctuations and response functions, such as the isobaric specific heat $C_{P}$ or the magnitude of the isobaric thermal expansion coefficient $\alpha_{P}$, largely increase when temperature is decreased [2]. These anomalies are more pronounced in the supercooled liquid state, below $0^{\circ} \mathrm{C}$. This state, metastable with respect to ice, can be observed at temperatures as low as $-47^{\circ} \mathrm{C}$ in plants $3,-41^{\circ} \mathrm{C}$ in laboratory at atmospheric pressure [4] and $-92^{\circ} \mathrm{C}$ at 2 kbar [5].

The anomalies have been interpreted on the basis of models that propose different scenarios. The scenarios can be divided in two main categories: $(a)$ those scenarios that include the coexistence at low $T$ of two liquids with different densities, and $(b)$ a scenario in which water forms local regions of different densities, but does not separate into two phases.

\subsection{Scenarios with coexistence of two liquids}

The liquid-liquid critical point scenario. In 1992 Poole et al. [6], on the basis of molecular dynamic simulations for ST2- and TIP4P-water, proposed that supercooled water separates into two liquid phases with different densities below $200 \mathrm{~K}$ and above $150 \mathrm{MPa}$. The low-density liquid (LDL) form of water and the high-density liquid (HDL) coexist along a first-order phase separation line with negative slope in the pressuretemperature $P-T$ phase diagram and terminate in a liquid-liquid critical point (LLCP). The LLCP scenario has been confirmed by simulations with various water models [7].

The occurrence of the LLCP has been rationalized with different theoretical models by assuming (a) the anticorrelation between energy and volume in the $\mathrm{H}$ (hydrogen) bonds formation and (b) the possibility of forming different kinds of $\mathrm{H}$ bonds. An example is the thermodynamic free energy model of Poole et al. that hypothesizes the formation of strong $\mathrm{H}$ bonds when a geometrical condition on the molar volume is satisfied [8].

Another example is the microscopic cell model introduced in Ref. [9, 10] and studied in detail in Ref. [11, 12, in which the correlation between the $\mathrm{H}$ bonds, due to the O$\mathrm{O}-\mathrm{O}$ interaction, is incorporated. In this model the liquid-liquid (LL) phase transition is due to the tetrahedral ordering at low $T$ and $P$ of the $\mathrm{H}$ bonds, as explained in the following section.

The LLCP is predicted to lie in a region of the $P-T$ phase diagram where the freezing of bulk water is inevitable. Therefore, direct experimental verification of the LLCP scenario is challenging. However, Soper and Ricci in 2000 showed with neutron diffraction measurements that the local arrangement of water molecules changes up to the second shell, increasing the local density when $P$ is increased from $26 \mathrm{MPa}$ to $400 \mathrm{MPa}$ at $-5.15^{\circ} \mathrm{C}$ 13. This structural change was initially observed by varying $T$ from about $263 \mathrm{~K}$ to $313 \mathrm{~K}$ in x-ray structure factor experiments for heavy water $\mathrm{D}_{2} \mathrm{O}$ in 1983 [14. The data show that by decreasing $T$ the average O-O-O angle increases 
Pressure Effects in Supercooled Water: Comparison between a 2D Model of Water and Experiments for Surf toward the tetrahedral angle $109.47^{\circ}$ [14]. This result has been reaffirmed by Ricci et al. in a recent experiment [15].

The critical point free scenario. Another scenario that hypothesizes a liquid-liquid (LL) phase transition has been considered recently [16. In this scenario the LL first order phase transition extends to negative $P$ and merges the liquid spinodal, but without a critical point. A rationalization of this scenario has been recently proposed on the basis of the microscopic cell model for water [9, 17, showing that this scenario has a liquid spinodal that reenters from negative to positive $P$ [17].

The stability limit scenario. The reentrant spinodal discussed in Ref. [17] was earlier proposed in the stability limit scenario [18] as the origin of the anomalies of water. Although in the initial formulation of the stability limit scenario the occurrence of the LL phase transition was not hypothesized, it was successively introduced for thermodynamic consistency [19] and found also in the thermodynamic free energy model of Poole et al. [8].

\subsection{Scenario without coexistence of two liquids}

The singularity free scenario. This scenario assumes that the H-bonds linking molecules are uncorrelated. Under this hypothesis, water anomalies are the effect of the negative volume-entropy cross fluctuations [20, 21] and the large increase of response functions seen in the experiments represents only an apparent singularity, due to local density fluctuations. The regions with different local density do not form separate phases. A pressure increase gives rise to a sharp, but continuous, increase of density, as in the supercritical region of the LLCP scenario. The continuous structural change is found also in $a b$ initio water simulations [22] at very high pressure, $P=10^{4} \mathrm{MPa}$, and $T=600 \mathrm{~K}$. This scenario is recovered by the microscopic cell model [9] in the limiting case of no cooperativity among the $\mathrm{H}$ bonds.

The singularity-free scenario, in the region accessible by experiments, reproduces the same phase diagram as the scenarios with coexistence of two liquids. Therefore, it is interesting to understand if there are oberservable differences among these scenarios. In particular, recent experiments and simulations, described in the next sections, have analyzed the case of the dynamics of water surrounding proteins or confined in nanopores, interpreting the experimental results within the context of the different scenarios.

\section{Hydrated proteins}

Recent experiments on surface water and water hydrating proteins [23, 24, 25] have shown that liquid water exists at temperatures as low as $-113^{\circ} \mathrm{C}[26$ ] at ambient pressure. At these extremely low temperatures interesting dynamical phenomena 
Pressure Effects in Supercooled Water: Comparison between a $2 D$ Model of Water and Experiments for Surf

occur [27, 28, 29, 30], suggesting a possible relation for the dynamics of the biological macromolecules with that of the surrounding water [25, 31].

At low $T$, proteins exist in a ("glassy") state with no conformational flexibility and with very low biological activity. For hydrated proteins above about $220 \mathrm{~K}$, the flexibility is restored, allowing the sampling of more conformational sub-states. Hence, the protein becomes biologically active at these temperatures. This dynamical transition is common to many biopolymers and is believed to be triggered by the strong coupling with the mobility of the hydration water [28], which shows a similar dynamical transition at about the same $T$ [23]. Chen et al. by studying the translational correlation time of water molecules hydrating a lysozyme protein [23], DNA [32] and RNA [33], found that at about $220 \mathrm{~K}$ the dynamics of hydration water changes from non-Arrhenius at high $T$ to Arrhenius at low $T$. By definition a correlation time $\tau$ has an Arrhenius behavior when

$$
\tau=\tau_{0} \exp \left[E_{\mathrm{A}} / k_{B} T\right]
$$

where $\tau_{0}$ is the correlation time in the high- $T$ limit, $E_{\mathrm{A}}$ is a $T$-independent activation energy and $k_{B}$ is the Boltzmann constant. On the other hand, $\tau$ is non-Arrhenius when its behavior cannot be fitted with the expression in Eq. (1).

Motivated by these experiments, Kumar et al. [34] simulated using the TIP5P model the dynamics and thermodynamic behavior of hydration water for (i) an orthorhombic form of hen egg-white lysozyme and (ii) a Dickerson dodecamer DNA at constant pressure $P=1 \mathrm{~atm}$, several constant temperatures $T$, and constant number of water molecules $N$. Kumar et al. [34 found that the mean square fluctuations $\left\langle x^{2}\right\rangle$ of the biomolecules changes its functional form below $T_{\mathrm{p}} \approx 245 \mathrm{~K}$, for both lysozyme and DNA. They also found that the specific heat $C_{P}$ of the total system (biopolymer and water) displays a maximum at $T_{\mathrm{W}} \approx(250 \pm 10) \mathrm{K}$ for both biomolecules.

To describe the quantitative changes in structure of hydration water, Kumar et al. [34] calculated the local tetrahedral order parameter $Q$ [35] for hydration water surrounding lysozyme and DNA and found that the rate of increase of $Q$ has a maximum at $T_{\mathrm{Q}}=(245 \pm 10) \mathrm{K}$, the same temperature of the crossover in the behavior of mean square fluctuations. Finally, they found that the diffusivity of hydration water exhibits a dynamic crossover from non-Arrhenius to Arrhenius behavior at the crossover temperature $T_{\times} \approx(245 \pm 10) \mathrm{K}$ for lysozyme and $T_{\times} \approx(250 \pm 10) \mathrm{K}$ for DNA. Note that $T_{\times}$is much higher than the glass transition temperature, estimated for TIP5P as $T_{g}=215 \mathrm{~K}$ [7]. Thus this crossover is not likely to be related to the glass transition in water. Therefore, the fact that $T_{\mathrm{p}} \approx T_{\times} \approx T_{\mathrm{W}} \approx T_{\mathrm{Q}}$ is evidence of the correlation between the changes in protein fluctuations and the hydration water thermodynamics and structure. Before analyzing in more details this relation, is worth considering the results of experiments about water confined in nanostructures. 
Pressure Effects in Supercooled Water: Comparison between a 2D Model of Water and Experiments for Surf

\section{Confined water}

The non-Arrhenius to Arrhenius dynamic crossover has been found also in water confined in $20 \AA$ MCM-41 silica pores. In 2004, indeed, Faraone et al. found the crossover for confined water at $T_{L} \approx 221 \mathrm{~K}$ by studying the structural relaxation time by neutron scattering [36]. This result was reinforced by the neutron magnetic resonance measurements of Mallamace et al. showing the crossover at $T_{L} \approx 225 \mathrm{~K}$ for the self diffusion of confined water [26.

In 2005, Liu et al. [37] showed that, by increasing the pressure, the dynamic crossover of water confined in $20 \AA$ MCM- 41 silica pores disappears at pressure between 1200 and 1600 bar. Xu et al. [38], by using simulations of TIP5P, ST2 water and other models for liquids with the LLCP, showed that the disappearing of the dynamic crossover of water can be associated to the presence of the LLCP $\left(C^{\prime}\right)$. The simulations for water, indeed, display the same phenomenology of the experiments, with a crossover in the diffusion coefficient at $P$ below the LL critical pressure $P<P_{C^{\prime}}$, and with no crossover at $P>P_{C^{\prime}}$. Xu et al. 38 presented numerical evidences that the crossover at $P<P_{C^{\prime}}$ is associated to the structural change occurring at the temperature of the maximum of the specific heat $C_{P}^{\max }$ along a line departing from the LLCP and extending in the one-phase region of the $P-T$ phase diagram. This line, close to the LLCP, coincides with the Widom line [12], defined as the locus of the maximum correlation length in the one-phase region.

$\mathrm{Xu}$ et al. 38] interpreted the absence of the crossover in the diffusion coefficient at $P>P_{C^{\prime}}$ as a consequence of the fact that the HDL-to-LDL spinodal occurs at almost constant $P \simeq P_{C^{\prime}}$ for decreasing $T$. Therefore, when cooled at $P>P_{C^{\prime}}$, water never crosses the HDL-to-LDL spinodal and does not undergo the structural change responsible for the dynamic crossover.

Hence, although the origin of the crossover has different interpretations [29], the experimental and numerical evidences suggest that the change in the dynamics is triggered by a local rearrangement of the $\mathrm{H}$ bond network [30]. Experiments and simulations, however, cannot give a definitive answer due to their finite resolution. For this reason is interesting to analyze the dynamic crossover of supercooled water within the framework of a Hamiltonian cell model that allows simulations and analytic calculations [10, 12].

\section{Water model}

We consider a cell model for water [9, 10, 11, 12] based on the experimental observations that on decreasing $P$ at constant $T$, or on decreasing $T$ at constant $P$, (i) water displays an increasing local tetrahedrality [39, 40], (ii) the volume per molecule increases at sufficiently low $P$ or $T$, and (iii) the O-O-O angular correlation increases [13, 15], consistent with simulations [22, 41].

The system is divided into cells $i \in[1, \ldots, N]$ on a regular lattice, each containing a 
Pressure Effects in Supercooled Water: Comparison between a 2D Model of Water and Experiments for Surf

molecule, with a volume $v_{i} \geq v_{0}$, where $v_{0}$ is the hard-core volume of one molecule, with a total volume $V=\sum_{i}^{N} v_{i}$. In $d$ dimensions the distance between two nearest neighbor (n.n.) molecules $i$ and $j$ is $r_{i, j} \equiv\left(v_{i}^{1 / d}+v_{j}^{1 / d}\right) / 2$. Since $v_{i}$ is a continuous variable, the distance $r_{i, j}$ is continuous.

Every cell is occupied by a molecule. The dimensionless density for the molecule in cell $i$ is $v_{0} / v_{i} \in(0,1]$. We use a discrete two-state liquid-index $n_{i}$ to quantify if the cell $i$ is in the liquid phase or not, with $n_{i}=1$ if $v_{0} / v \geq 0.5$ and $n_{i}=0$ otherwise. Therefore, $\sum_{i}^{N} n_{i}$ is the total number of liquid cells and $N-\sum_{i}^{N} n_{i}$ is the total number of gas cells.

The van der Waals attraction between the molecules is represented by the Hamiltonian term

$$
\mathcal{H} \equiv-\epsilon \sum_{\langle i, j\rangle} n_{i} n_{j}
$$

where $\epsilon>0$ is the van der Waals attraction energy, which induces the liquid-gas phase transition.

Each molecule $i$ has four H-bond indices $\sigma_{i j} \in[1, \ldots, q]$, corresponding to four n.n. cells $j$, giving rise to $q^{4}$ different molecular orientations. Bonding and intramolecular (IM) interactions are accounted for by, respectively, the two Hamiltonian terms

$$
\mathcal{H}_{\mathrm{B}} \equiv-J \sum_{\langle i, j\rangle} n_{i} n_{j} \delta_{\sigma_{i j} \sigma_{j i}}
$$

where the sum is over n.n. cells, $0<J<\epsilon$ is the bond energy, $\delta_{a, b}=1$ if $a=b$ and $\delta_{a, b}=0$ otherwise, and

$$
\mathcal{H}_{\mathrm{IM}} \equiv-J_{\sigma} \sum_{i} n_{i} \sum_{(k, \ell)_{i}} \delta_{\sigma_{i k} \sigma_{i \ell}}
$$

where $\sum_{(k, \ell)_{i}}$ denotes the sum over the IM bond indices $(k, l)$ of the molecule $i$ and $J_{\sigma}>0$ is the IM interaction energy with $J_{\sigma}<J$, which models the angular correlation between the bonds on the same molecule.

When $\mathrm{H}$ bonds are formed, a small volume $v_{\mathrm{B}}$ is added to the local volumes $v_{i}$ and $v_{j}$ of the two H-bonded molecules $i$ and $j$, increasing their average distance to $r_{i j} \equiv\left(v_{i}+v_{j}+v_{\mathrm{B}}\right)^{1 / d} / 2$. Pictorially, this can be viewed as a local increase of the excluded volume associated with molecules $i$ and $j$ and is consistent with the experimental observation that H-bonded molecules form a low density open structure. Therefore, the total volume is proportional to the total number $N_{\mathrm{B}}$ of $\mathrm{H}$ bonds, as

$$
V \equiv V_{0}+N_{\mathrm{B}} v_{\mathrm{B}}
$$

where $V_{0} \equiv N v_{0}$ is the volume of the liquid with no $\mathrm{H}$ bonds, and

$$
N_{\mathrm{B}} \equiv \sum_{\langle i, j\rangle} n_{i} n_{j} \delta_{\sigma_{i j}, \sigma_{j i}} .
$$

Therefore, the cell model has the total Hamiltonian

$$
\mathcal{H}_{\text {tot }} \equiv \mathcal{H}+\mathcal{H}_{\mathrm{B}}+\mathcal{H}_{\mathrm{IM}}
$$

with the volume given by Eq. (5). 
Pressure Effects in Supercooled Water: Comparison between a 2D Model of Water and Experiments for Surf

\section{Thermodynamics of the water model}

The cell model described in the previous section has been analyzed by mean field calculations [9, 10, 11, 12, 42, 43] and Monte Carlo simulations [11, 42, 43]. The model's parameters considered in these studies are: $J / \epsilon=0.5, J_{\sigma} / \epsilon=0.05, v_{\mathrm{B}} / v_{0}=0.5$ and $q=6$.

\subsection{Mean field}

The mean field results [12], consistent with computer simulations [11], reproduce the known phase diagram of fluid water, with the liquid-gas coexistence region ending in the critical point $C$, at $k_{B} T_{C} / \epsilon=1.03 \pm 0.03$ and $P_{C} v_{0} / \epsilon=0.18 \pm 0.04$, and with the temperatures of maximum density (TMD) at constant $P$ that decreases with increasing $P$ as in the experiments [49, 50].

In the deeply supercooled region the density has another discontinuity marking the coexistence region between two liquids at different densities. This discontinuity is associated with a discontinuity in $m_{\sigma}^{\min }$, the value of the tetrahedral order parameter $m_{\sigma} \in[0,1]$ that minimizes the molar Gibbs free energy $g$. Here, $m_{\sigma}$ quantifies the number of $\mathrm{H}$ bonds with tetrahedral orientation and is defined as the difference between the number-density of $\sigma_{i j}=1$ and the average number-density for the other $q-1$ states, i.e., $m_{\sigma} \equiv n_{\sigma}-\left(1-n_{\sigma}\right) /(q-1)$, where

$$
n_{\sigma} \equiv \frac{1}{4 n N} \sum_{\langle i, j\rangle} n_{i} n_{j} \delta_{\sigma_{i j}, 1}
$$

and where the state $\sigma_{i j}=1$ corresponds to the appropriate state to form a (not bifurcated) H-bond with local tetrahedral order.

In the $N P T$ ensemble, the relevant free energy is the Gibbs energy per mole

$$
g \equiv u-T s+P v,
$$

where $u$ is the molar energy, $s$ the molar entropy, and $v$ the molar volume. As explained in Ref. [12], these quantities can all be written in the mean field approximation, giving rise to a mean field expression for $g$ that can be minimized with respect to the gas-liquid order parameter $m$ and the tetrahedral order parameter $m_{\sigma}$.

For any $P$ at low $k_{B} T / \epsilon<0.1, g$ has its minimum for $m=1$ (liquid-phase value) and for a value $m_{\sigma}^{\min }$ that changes with $T$ and $P$ [Fig.(1)]. At constant $P, m_{\sigma}^{\min }$ decreases with increasing $T$, displaying a discontinuity above $P v_{0} / \epsilon \simeq 0.8$ [Fig.(11) right]. The discontinuity disappears at $P v_{0} / \epsilon \simeq 0.8$ [Fig. (1) center] and the approach of $m_{\sigma}^{\min }$ to 0 is always continuous below $P v_{0} / \epsilon=0.8$ [Fig. (1) left]. The appearance of the discontinuity in $m_{\sigma}^{\min }$ denotes the occurrence at high $P$ of a phase transition between two liquid phases with a different value of the tetrahedral order parameter $m_{\sigma}$ : an orientationally disordered phase $\left(m_{\sigma}=0\right)$ at high $T$, with no tetrahedral order, and a tetrahedrally ordered phase $\left(m_{\sigma}>0.5\right)$ at low $T$. The phase separation disappears at the liquidliquid critical point $C^{\prime}$, that in mean field is estimated at $k_{B} T_{C^{\prime}} / \epsilon=0.062 \pm 0.02$ and 
Pressure Effects in Supercooled Water: Comparison between a. श.D Mod.el of Water an.d. Frneriments for Surf
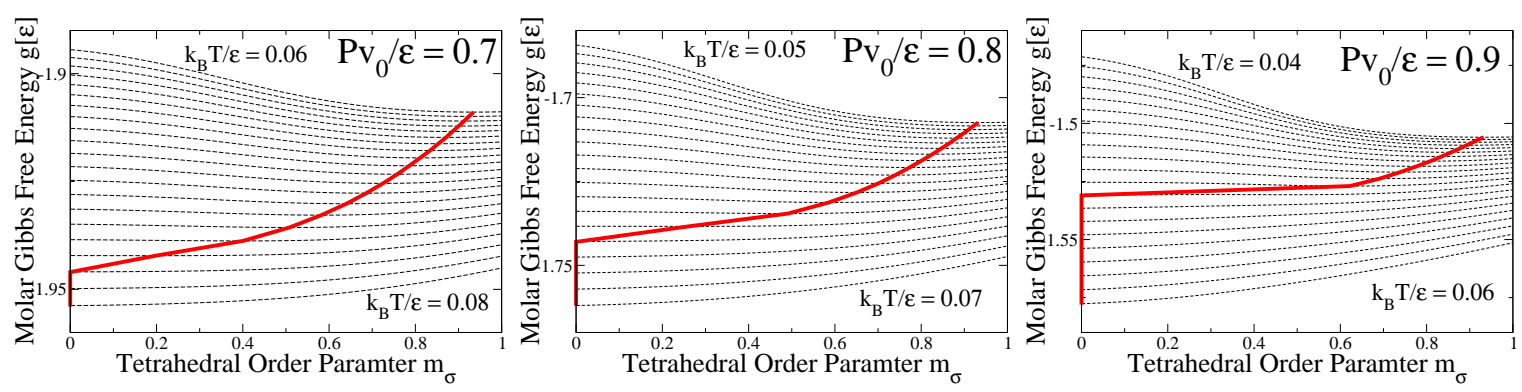

Figure 1. The mean field molar Gibbs free energy $g$ (dashed lines), in units of $\epsilon$, as function of the dimensionless tetrahedral order parameter $m_{\sigma} \in[0,1]$ for different choices of $T$ and $P$. The thick (red) line connects the points $m_{\sigma}^{\min }$ of minimum $g$ at different $T$ for $P v_{0} / \epsilon=0.7$ (left panel), 0.8 (center panel), 0.9 (right panel). In each panel the topmost line corresponds to the lowest $k_{B} T / \epsilon(0.06,0.05$ and 0.04 , respectively) and the bottom line to the highest $k_{B} T / \epsilon(0.08,0.07,0.06$, respectively) with lines separated by $k_{B} \delta T / \epsilon=0.001$. In all the panels $m_{\sigma}^{\min }$ increases when $T$ decreases, being 0 at the higher temperatures and $\simeq 0.9$ at the lowest temperature. The value $m_{\sigma}=0$ corresponds to the absence of tetrahedral order, i.e. to the high-density arrangement of water molecules. The value $m_{\sigma}=1$ corresponds to full tetrahedral order of the $\mathrm{H}$ bonds, i.e. to the low-density arrangement of the water molecules. At $P v_{0} / \epsilon=0.7$ (left panel), by increasing $T, m_{\sigma}^{\min }$ changes without discontinuity from $\simeq 0.9$ at $k_{B} T / \epsilon=0.06$ to 0 at $k_{B} T / \epsilon=0.078$, denoting a continuous change from the low-density arrangement at low $T$ to the high-density arrangement at high $T$. At $P v_{0} / \epsilon=0.9$ (right panel), instead, by increasing $T, m_{\sigma}^{\min }$ changes with discontinuity from $\simeq 0.6$ at $k_{B} T / \epsilon=0.051$ to 0 at $k_{B} T / \epsilon=0.052$, denoting a discontinuous phase change from the low-density liquid (LDL) to the high-density liquid (HDL). The pressure $P v_{0} / \epsilon=0.8$ (center panel) is very close to the critical pressure $P_{C^{\prime}}$, below which the discontinuity seen at higher $P$ disappears.

$P_{C^{\prime}} v_{0} / \epsilon=0.82 \pm 0.02$ [12]. The discontinuity in $m_{\sigma}$ determines the discontinuity in the density between the two phases, as can be shown analytically [12], separating the liquid in LDL at low $T$ and HDL at high $T$, consistent with the LLCP scenario.

\subsection{Monte Carlo}

In the Monte Carlo simulations of the cell model, the Hamiltonian $\mathcal{H}_{\text {tot }}$ in Eq.(17) is simulated with the standard spin-flip method [11, 42, 43]. For sake of simplicity, we solve the model under the condition of being in a homogeneous phase with $v_{i}=v$ for any $i$. Although this condition can be easily removed, the solution of the model under this condition gives good qualitative agreement with the experiments, as we will see in the following sections.

The model is defined in any dimension. However, since we assume for simplicity that any molecule can form at most four $\mathrm{H}$ bonds, we solve the model on a regular square lattice of cells. This choice is particularly appropriate for the study of quasi-2D water [44] between hydrophobic surfaces and is a first order approximation to the layer of water between the surface of a protein and the surface of the frozen bulk water at low $T$. Of course, in this approximation the interaction with the confined surfaces is not 
taken into account [45]. Nevertheless, the qualitative comparison with the experiments is satisfactory. The lack of the third dimension, and the number of neighbors limited to four, could affect properties related to the amount of free space around a molecule, such as the diffusion constant [46, 47, 48]. These properties are not considered in the analysis of the model reported here.

To further simplify the simulations, the van der Waals interaction, represented in mean field by the Hamiltonian term in Eq.(2), is replaced by an equivalent LennardJones potential with characteristic energy $-\epsilon$ at distance $R_{0} \equiv v_{0}^{1 / d}$ and truncated with a hard-core in its minimum at $R_{0}$ [11, 42, 43. Simulations performed with a standard Lennard-Jones potential, without truncation, show that the results are qualitatively unchanged [47].

The resulting phase diagram [11] displays, at high $T$ and low $P$, a gas-liquid phase transition ending in a gas-liquid critical point $C$. From $C$ departs in the supercritical region the gas-liquid Widom line, i.e. the line of maximum - but finite - correlation length for the fluid. At lower $T$, the phase diagram displays the TMD line, retracing toward low $T$ at high $P$ as in the experiments 3. At lower $T$ and high $P$, the phase diagram displays a HDL-LDL phase transition with negative slope in the $P-T$ plane, ending in a LL critical point $C^{\prime}$. As for the gas-liquid critical point, also from $C^{\prime}$ the line of maximum correlation length for the liquid, the LL Widom line [38, 12], departs into the one-phase region (Fig. 2). Therefore, the Monte Carlo results confirm the mean field analysis, displaying a phase diagram consistent with the LLCP scenario.

Recent calculations [17] show that by varying the parameters of the cell model, it is possible to recover also $(i)$ the singularity free scenario, $(i i)$ the stability limit scenario and $($ iii) the critical point free scenario. In the following we report about the analysis of the dynamics of the model for the LLCP scenario and the singularity free (SF) scenario.

\section{Dynamics of the water model}

We first consider the case of the LLCP scenario. Performing Monte Carlo simulations for $T$ and $P$ around the values of the LL Widom line $T_{W}(P)$, Kumar et al. [42 found that the correlation time $\tau$ of $S_{i} \equiv \sum_{j} \sigma_{i j} / 4$, which quantifies the degree of total bond ordering for site $i$, displays a dynamic crossover from Vogel-Fulcher-Tamman (VFT) function at high $T$ to Arrhenius $T$-dependence at low $T$ (Fig. 3,a). The VFT function is given by

$$
\tau^{\mathrm{VFT}} \equiv \tau_{0}^{\mathrm{VFT}} \exp \left[\frac{T_{1}}{T-T_{0}}\right]
$$

where $\tau_{0}^{\mathrm{VFT}}, T_{1}$, and $T_{0}$ are three fitting parameters, and the Arrhenius function is given in Eq.(1). This result is qualitatively consistent with the dynamic crossover found in experiments for confined water and hydration water [23, 26, 32, 33, 36, 37] and has been related to the presence of the LLCP [38, 34].

It is, therefore, interesting to compare this result with the dynamic behavior of the cell model in the hypothesis in which the SF scenario holds. To do this, Kumar et 


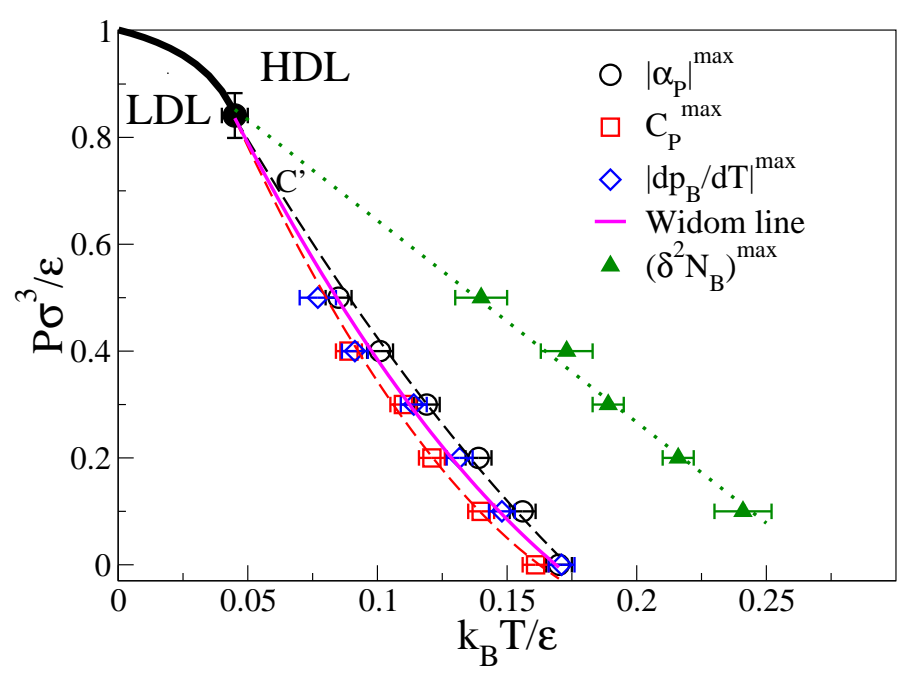

Figure 2. The Monte Carlo phase diagram for the cell model at low $T$ for $N=3600$ water molecules [43. $C^{\prime}$ is the HDL-LDL critical point, end of first-order phase transition line (thick line) 9]; symbols are maxima of the coefficient of thermal expansion $\left|\alpha_{P}\right|^{\max }(\bigcirc)$, isobaric specific heat $C_{P}^{\max }(\square),\left|d p_{\mathrm{B}} / d T\right|^{\max }(\diamond)$ the numerical derivative of the probability of forming a H-bond, proportional to the fluctuation of the number of bonds $\left(\delta^{2} N_{\mathrm{B}}\right)^{\max }(\triangle)$; the Widom (solid) line, corresponding to locus of maximum correlation length and estimated as the average between $\left|\alpha_{P}\right|^{\max }$ and $C_{P}^{\max }$, coincides within the error bars with $\left|d p_{\mathrm{B}} / d T\right|^{\text {max }}$, i.e. with the locus of the maximum structural variation. Dashed lines are guide for the eyes.

al. [42] analyzed the dynamics of the cell model when the H-bond correlation is zero $\left(J_{\sigma}=0\right)$ and the cell model recovers the SF model of Sastry et al. [20].

The result (Fig. 3, b) [42] shows that also in this scenario a dynamic crossover is expected. The temperature of the crossover is coinciding, within the numerical precision, with the temperature $T\left(C_{P}^{\max }\right)$ of maximum isobaric specific heat, which in the $\mathrm{SF}$ scenario play a role equivalent to $T_{W}(P)$ of the LLCP scenario. Indeed, both $T\left(C_{P}^{\max }\right)$ and $T_{W}(P)$ mark the temperature $T_{\text {struct }}^{\max }$ of the maximum structural change for the liquid [43].

The cell model allows to clarify that the dynamic crossover is, indeed, a direct consequence of the structural change occurring at $T_{\text {struct }}^{\max }$. By calculating, in the mean field approximation, the $T$-dependent activation energy $E_{\mathrm{A}}(T)^{\mathrm{MF}}$ necessary to $(i)$ break a non-tetrahedral $\mathrm{H}$ bond, (ii) reorient the water molecule and (iii) form a new $\mathrm{H}$ bond in a tetrahedral orientation, Kumar et al. [43] calculated the mean field correlation time as

$$
\tau^{\mathrm{MF}}=\tau_{0} \exp \left[\frac{E_{\mathrm{A}}(T)^{\mathrm{MF}}}{k_{B} T}\right]
$$

and compared $\tau^{\mathrm{MF}}$ with the correlation time from the Monte Carlo simulations, finding an excellent agreement. Since $E_{\mathrm{A}}^{\mathrm{MF}}$ is an explicit function of the number $N_{\mathrm{B}}$ of $\mathrm{H}$ bonds, therefore $\tau^{\mathrm{MF}}$ is a function of the structure of the liquid [43]. Since $N_{\mathrm{B}}$ rapidly changes for $T>T_{\text {struct }}^{\max }$, has its maximum variation at $T_{\text {struct }}^{\max }$, and slowly changes for 
Pressure Effects in Supercooled Water: Comparison between a 2D Model of Water and Experiments for Surf
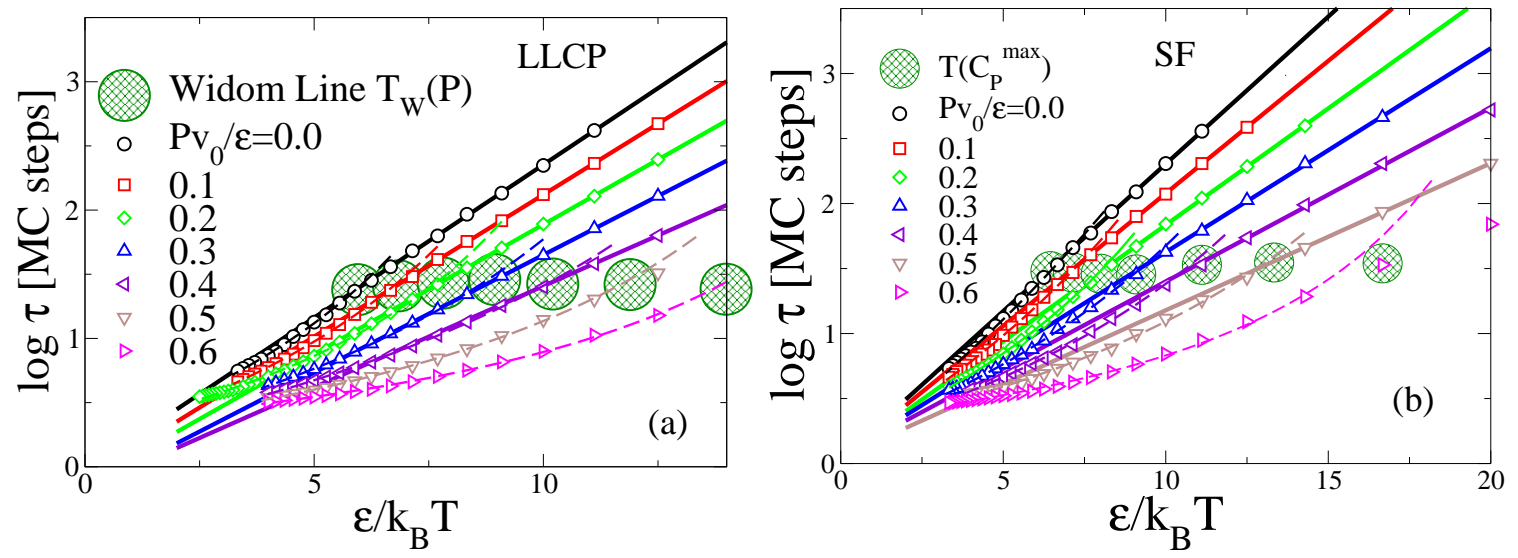

Figure 3. Monte Carlo results for the dynamic crossover in the orientational relaxation time $\tau$ of the cell water model for a range of different pressures [42. (a) In the case of the liquid-liquid critical point (LLCP) scenario, the crossover occurs at a temperature consistent with the Widom line $T_{W}(P)$. (b) In the case of the singularity free (SF) scenario, the crossover occurs at a temperature consistent with the $T\left(C_{P}^{\max }\right)$. In both panels the large hatched circles mark $T_{W}(P)$ and $T\left(C_{P}^{\max }\right)$, with a radius approximately equal to the error bar. Solid and dashed lines represent Arrhenius and Vogel-FulcherTamman fits, respectively. In both cases, Kumar et al. 42] predicted that the dynamic crossover occurs at approximately the same value of $\tau$ for all the values of pressures studied, i.e. the crossover is isochronic.

$T<T_{\text {struct }}^{\max }$, the correlation time is a non-Arrhenius function of $T$ for $T>T_{\text {struct }}^{\max }$ and almost an Arrhenius function for $T<T_{\text {struct }}^{\max }$, giving rise to a dynamic crossover [43]. The agreement between the mean field calculation and the Monte Carlo results clarifies that the low- $T$ regime is only approximately Arrhenius.

The analysis of the cell model allows, furthermore, to make a number of predictions about the dynamics of water at low $T$.

(i) At the dynamic crossover the value of $\tau$ is about the same for all the pressures considered in Ref. [42], i.e. the crossover is isochronic and the correlation time at the crossover is $\tau_{\mathrm{L}}=10^{3 / 2}$ in units of Monte Carlo steps [42]. Comparison with experiments, in the next section, allows to convert this result in real time units.

(ii) The activation energy $E_{\mathrm{A}}$, that is found by fitting the low- $T$ dynamic regime with an Arrhenius behavior, decreases linearly for increasing $P$ [42].

(iii) If we fix a characteristic time, for example the crossover time $\tau_{\mathrm{L}}$, the temperature $T_{\mathrm{L}}$ at which this correlation time is reached decreases linearly for increasing $P$ [42].

All these predictions are verified in both the LLCP scenario and the SF scenario. However, Kumar et al. 42] found a difference between the two scenarios: for the LLCP scenario the index $E_{\mathrm{A}} /\left(k_{B} T_{\mathrm{L}}\right)$ increases for increasing $P$, while for the SF scenario $E_{\mathrm{A}} /\left(k_{B} T_{\mathrm{L}}\right)$ is constant. However, the predicted increase of $E_{\mathrm{A}} /\left(k_{B} T_{\mathrm{L}}\right)$ is of the order of $1 \%$ [42]. As we will discuss in the next section, this increase is within the present experimental error bar. 
Pressure Effects in Supercooled Water: Comparison between a $2 D$ Model of Water and Experiments for Surf

\section{Comparison with the experiments on hydration water}

Recently, Chen and coworkers have performed extensive QENS experiments to investigate the dynamical behavior of hydration water on lysozyme, spanning a range of pressures going from ambient pressure up to 1600 bar [51]. By measuring the mean square displacement of the of $\mathrm{H}$-atoms in lysozyme, they found a dynamic crossover for all pressures studied [51]. They also found that the translational correlation time of the H-atoms of the hydration water shows a dynamic crossover for $P=1,400,800,1200$, 1500 bar [51]. For $P \leq 1500$ bar, they showed that the lysozyme dynamic crossover and the hydration water dynamic crossover occurs at the same $T$ [51], extending their previous results on lysozyme [23], DNA [32] and RNA [33] at ambient pressure.

They also showed that the dynamic crossover for the hydration water disappears at $P=1600$ bar [51]. This finding, together with their previous measurements for water confined in MCM-41 Silica pore [37], suggests that the disappearing of the nonArrhenius to Arrhenius crossover at $P=1600$ bar is independent of the constraint used to avoid homogeneous ice nucleation. As shown in Ref. [38], the disappearing of the crossover could be the consequence of the LLCP occurring at a pressure between 1500 and 1600 bar.

The comparison of the experimental data in Ref. [51] with the results of the Monte Carlo simulations allows several observations.

- The correlation time $\tau_{\mathrm{L}}$ at the crossover is constant as predicted by the cell model, point (i) in the previous section.

- $\tau_{\mathrm{L}}=10^{3 / 2}$ in units of Monte Carlo steps (MCS) 42] corresponds to $10^{4.25} \mathrm{ps}$, i.e. $1 \mathrm{MCS} \approx 600$ ps (Fig. (4).

- The high- $T$ (non-Arrhenius) behavior of the correlation time in real water has a stronger dependence on $T$ than the one seen in the cell model at $P=0$ (Fig. (4) and is more similar to the behavior seen in the model at higher $P$. Since in the model this behavior is regulated by the increase of number $N_{\mathrm{B}}$ of $\mathrm{H}$ bonds for decreasing $T$, this observation implies that in real water $N_{\mathrm{B}}$ rapidly increases approaching the temperature of maximum structural change $T_{\text {struct }}^{\max }$, and $N_{\mathrm{B}}$ is smaller in real water than in the model for $T>T_{\text {struct }}^{\max }$ [4].

- The low- $T$ (approximately Arrhenius) behavior is well reproduced by the cell model (Fig. (4). This result implies that the estimate of the activation energy at $P=0$ in the model compares with a reasonable agreement to the activation energy measured for hydration water.

- The direct comparison of the Monte Carlo estimated activation energy $E_{\mathrm{A}}$ and the QENS data (Fig. 5, upper panels) shows that the prediction (ii) of the previous section is verified in the experiments, with $E_{\mathrm{A}}$ decreasing linearly for increasing $P$. The agreement is only qualitative, since the variation of $E_{\mathrm{A}}$ in the cell model is too large with respect to the experiments. 
Pressure Effects in Supercooled Water: Comparison between a 2D Model of Water and Experiments for Surf

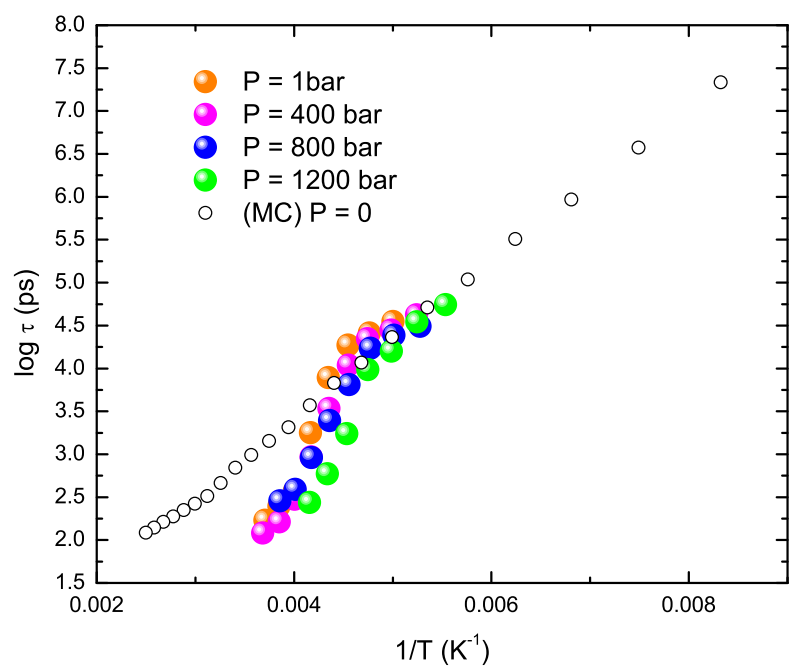

Figure 4. Comparison between the Monte Carlo [42] results and the QENS data [51] for the correlation time $\tau$ of supercooled water. Monte Carlo results (open symbols) are for $P=0$ and are rescaled in such a way that the crossover occurs at at the same $T$ and $\tau$ of the experimental data (full symbols). The experimental data are for pressures going from ambient to 1200 bar.

- The crossover temperature $k_{B} T_{\mathrm{L}}$ in the experiments verifies the prediction (iii) of the cell model, i.e. $k_{B} T_{\mathrm{L}}$ decreases linearly for increasing $P$ (Fig. 5, lower panels). Also in this case, the variation in the model is too large and the agreement is only qualitative.

- The crossover temperature $k_{B} T_{\mathrm{L}} / \epsilon \simeq 0.16$ at ambient pressure corresponds to $220 \mathrm{~K}$ (Fig. (4), leading to a van der Waals interaction strength $\epsilon \simeq 11 \mathrm{~kJ} \mathrm{~mol}^{-1}$ and a H-bond strength $J=0.5 \epsilon=5.5 \mathrm{~kJ} \mathrm{~mol}^{-1}$. This H-bond strength is about $1 / 4$ of the $\mathrm{H}$-bond strength expected below $100^{\circ} \mathrm{C}[52$ and is about the value estimated for then van der Waals attraction based on isoelectronic molecules at optimal separation [53].

We finally observe that the prediction of the model about the different behavior of the index $E_{\mathrm{A}} /\left(k_{B} T_{\mathrm{L}}\right)$ in the two scenarios, cannot be verified in the experiments, since the predicted difference between the two scenarios (of the order of $1 \%$ ) is within the error bars of the measurements [51]. The QENS data in Ref. [51] show a non-monotonic behavior for this index within an error bar larger than the $1 \%$ variation predicted for the LLCP scenario. Hence, the data could be considered also constant within the error bars, as predicted for the SF scenario. Therefore, is not possible to discriminate between the two scenarios on the basis of the present data for the index $E_{\mathrm{A}} /\left(k_{B} T_{\mathrm{L}}\right)$. 
Pressure Effects in Supercooled Water: Comparison between a 2D Model of Water and Experiments for Surf
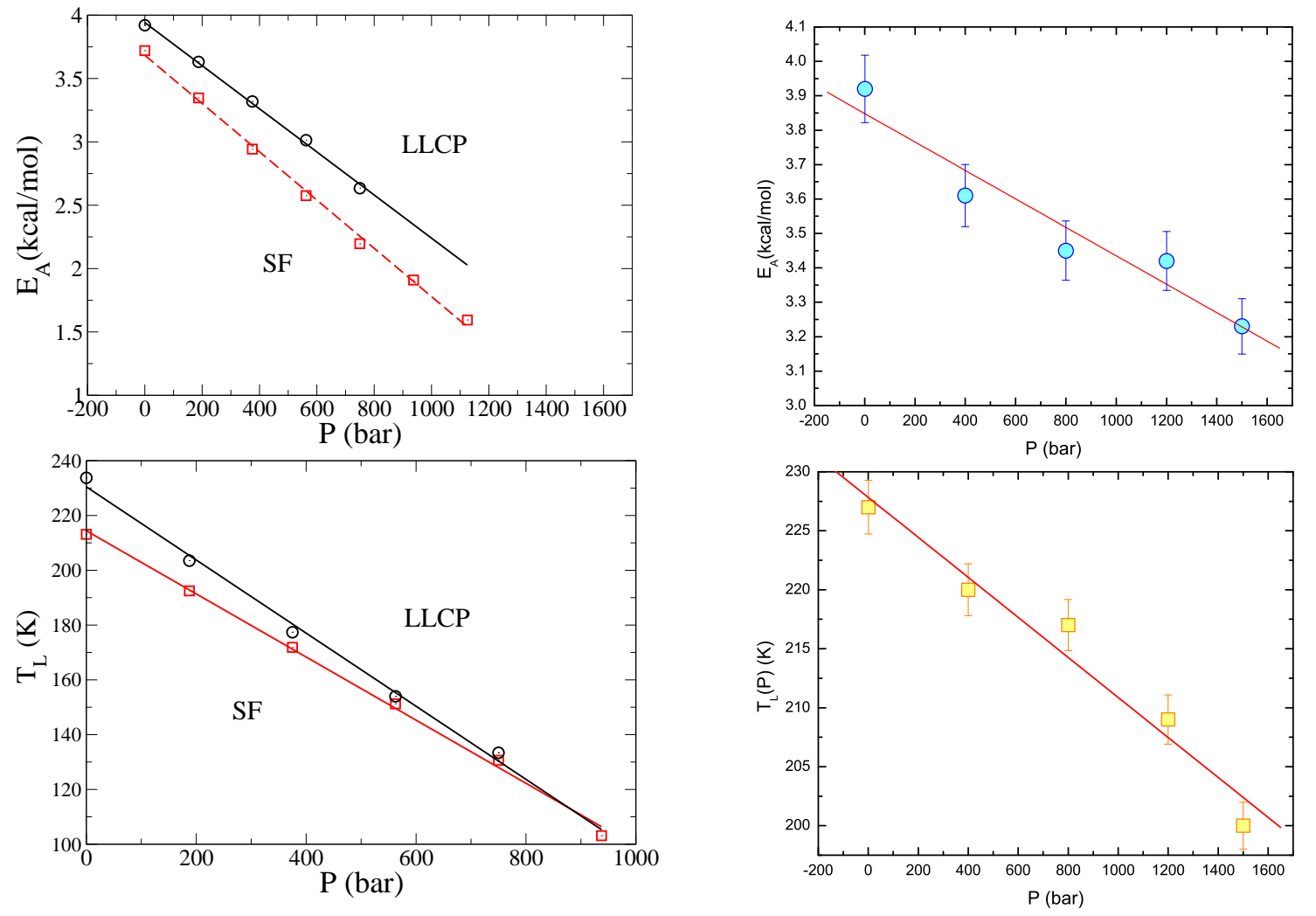

Figure 5. Monte Carlo [42] results and the QENS data [51] for the activation energy $E_{\mathrm{A}}$ of the low- $T$ regime and the crossover temperature $T_{\mathrm{L}}$, as functions of the pressure $P$. Upper left panel: Monte Carlo results for $E_{\mathrm{A}}$ [42]; for sake of comparison with the experimental data, pressure is rescaled by an arbitrary factor $1500 \mathrm{bar} /\left(0.8 \epsilon / v_{0}\right)$ and energy by a factor $16.4 \mathrm{kcal} \mathrm{mol}^{-1} / \epsilon \simeq 68.7 \mathrm{~kJ} \mathrm{~mol}^{-1} / \epsilon$; both the liquid-liquid critical point (LLCP) scenario results (circles) and the singularity free (SF) scenario results (squares) are reported. Lower left panel: Monte Carlo results for the crossover temperature $T_{\mathrm{L}}$; pressure is rescaled as above and temperature is rescaled by a factor $220 \mathrm{~K} /\left(0.16 \epsilon / k_{B}\right)$; symbols are as in previous panel. Upper right panel and lower right panel: QENS data for the lysozyme hydration water [51].

\section{Discussion and Conclusions}

Several questions remain open. The work reported here allows us to propose possible answers to some of these questions.

- Why does water have anomalies?

Simulations [6] first and, after, experiments [13] have shown the existence of two local configurations for water molecules: tetrahedral up to the second shell (or open) and non-tetrahedral (or closed). These local arrangements are the consequence of the orientational character of the $\mathrm{H}$ bonds and their typical competition between attraction and repulsion. Many of the anomalies can be understood on the basis of the tendency of the $\mathrm{H}$ bond to form open configurations to minimize the energy, frustrated by the necessity of reducing the occupied volume at high density or pressure. 
Pressure Effects in Supercooled Water: Comparison between a 2D Model of Water and Experiments for Surf

- What are the implications of these open and closed configurations?

The cell model discussed here [9, 10, 11, 12] shows that the existence of these local configurations, related to the tendency of the $\mathrm{H}$ bonds to correlate and order in a tetrahedral way, is enough to imply the occurrence of a liquid-liquid phase transition, possibly ending in a critical point. Only in the hypothesis that the $\mathrm{H}$ bonds formed by the same molecule are completely uncorrelated, as in the Sastry et al. model [20, the open and closed configurations do not separate into two phases, giving rise to the singularity free scenario.

- Why is there a dynamic crossover in the correlation time of supercooled water? Experiments [23] and simulations [38] offer evidences that the dynamic crossover is due to the local variation of $\mathrm{H}$ bond network [34]. The cell model discussed here, furthermore, offers an analytic relation, based on a mean field approximation, between the dynamic crossover and the structural change [42, 12]. In the cell model it is assumed that the relevant mechanism for the low- $T$ water dynamics is the breaking of the $\mathrm{H}$ bonds to reorient the molecule and form more tetrahedral bonds, as seen in simulations [54].

- Why is the dynamic crossover at the locus $T\left(C_{P}^{\max }\right)$ of maximum specific heat? At $T\left(C_{P}^{\max }\right)$, the number $N_{\mathrm{B}}$ of $\mathrm{H}$ bonds has its maximum variation. The liquid changes from the HDL-like local structure at high $T$ to LDL-like local structure at low $T$. In the HDL-like regions $N_{\mathrm{B}}$ rapidly increases when $T$ decreases, implying that the activation energy $E_{\mathrm{A}}$ for the breaking and reorientation of the $\mathrm{H}$ bonds increases and the behavior is non-Arrhenius [43]. In the LDL-like regions, $N_{\mathrm{B}}$ is almost constant with $T$, implying that $E_{\mathrm{A}}$ is approximately constant and the behavior is asymptotically Arrhenius [43].

- Is the dynamic crossover an evidence of the liquid-liquid critical point?

The dynamic crossover is consistent with both the liquid-liquid critical point scenario and the singularity free scenario [42]. However, in the hypothesis of the presence of a liquid-liquid critical point is possible to rationalize the disappearing of the dynamic crossover at high pressure [37, 38, 51], while in the other scenarios no change in the crossover is expected with the increase of $P$.

- Is the dynamic crossover a cooperative process?

The dynamic crossover is related to the increase of the correlation length $\xi$ in the liquid. This quantity increases for increasing $P$ along the Widom line and diverges at the liquid-liquid critical point. Therefore, the dynamic crossover is a cooperative process at the liquid-liquid critical point.

- How pressure studies can help us in understanding the physics of water?

The recent experiments on protein hydration water [51] show that is possible to analyze the dynamic crossover of water at very low $T$, measuring quantities, such as the crossover temperature $T_{\mathrm{L}}$ and the activation energy $E_{\mathrm{A}}$, whose behaviors have been predicted by the theory [42]. The experiments have verified three of the four theoretical predictions, being the forth within the error bars. 
Pressure Effects in Supercooled Water: Comparison between a 2D Model of Water and Experiments for Surf

In conclusion, in this report we compare the predictions of a cell model for water analyzed by $\mathrm{MC}$ in 2D [42] with the QENS data for water at the surface of lysozyme [51]. The data show a dynamic crossover from high- $T$ non-Arrhenius behavior to low- $T$ quasi-Arrhenius behavior for the water relaxation time at $P<1600$ bar. Both the temperature $T_{\mathrm{L}}(P)$ and the low- $T$ average activation energy $E_{\mathrm{A}}(P)$ linearly decrease for increasing $P$, as predicted by the model. The relaxation time at the crossover $\tau\left(T_{\mathrm{L}}\right)$ is independent of $P$, as in the model. It is an open question if this isochronic behavior is related to the constant-time dynamic crossover observed in glass formers [55].

The mean field approach [42, 43] allows to find a functional relation between $E_{\mathrm{A}}$ and the number $N_{\mathrm{B}}(T, P)$ of $\mathrm{H}$ bonds, i.e. allows to show in a clear way that the dynamic crossover is a consequence of the structural changes occurring at the temperature of maximum specific heat $T\left(C_{P}^{\max }\right)$. However, the comparison with the experiments does not clarify which scenario describes better the supercooled region of water. Indeed, within the experimental error bar larger than $1 \%$, both the SF and the LLCP scenario could be consistent. Nevertheless, the disappearing of the crossover observed in the experiments at $P=1600$ bar is expected only in the presence of a LLCP.

\section{Acknowledgments}

We thank our collaborators, M. I. Marqués, M. Yamada, and F. de los Santos, and the NSF Chemistry Program CHE 0616489 for support. G. F. also thanks the Spanish Ministerio de Ciencia e Innovación, project FIS2007-61433.

[1] P. Kumar, G. Franzese, S. V. Buldyrev, and H. E. Stanley, in Aspects of Physical Biology: Biological Water, Protein Solutions, Transport and Replication, G. Franzese and M. Rubi eds. (Springer, Berlin, 2008), p. 3; J. Swenson, H. Jansson, and R. Bergman, ibid., p. 23; F. Bresme, and A. Wynveen, ibid., p. 43; P. G. Debenedetti and H. E. Stanley, Physics Today 56, 40 (2003).

[2] C. A. Angell, J. Shuppert, and J. C. Tucker, J. Phys. Chem. 77, 3092 (1973); R. J. Speedy and C. A. Angell, J. Chem. Phys. 65, 851 (1976).

[3] P. G. Debenedetti, Metastable Liquids: Concepts and Principles (Princeton University Press, Princeton, 1996).

[4] B. M. Cwilong, Proc. Roy. Soc. A 190, 137 (1947).

[5] H. Kanno, R. J. Speedy, and C. A. Angell Science 189, 881 (1975).

[6] P. H. Poole, F. Sciortino, U. Essmann, and H. E. Stanley, Nature 360, 324 (1992); P. H. Poole, F. Sciortino, U. Essmann, and H. E. Stanley, Phys. Rev. E 48, 3799 (1993); P. H. Poole, U. Essmann, F. Sciortino, and H. E. Stanley, Phys. Rev. E 48, 4605 (1993).

[7] I. Brovchenko, A. Geiger, and A. Oleinikova, J. Chem. Phys. 118, 9473 (2003); I. Brovchenko, A. Geiger, and A. Oleinikova, J. Chem. Phys. 123, 044515 (2005).

[8] P. H. Poole et al., Phys. Rev. Lett. 73, 1632 (1994).

[9] G. Franzese and H. E. Stanley, J. Phys.-Cond. Mat. 14, 2201 (2002).

[10] G. Franzese and H. E. Stanley, Physica A, 314, 508 (2002).

[11] G. Franzese, M. I. Marqués, and H. E. Stanley, Phys. Rev. E 67, 011103 (2003).

[12] G. Franzese and H. E. Stanley, J. Phys.: Cond. Matt. 19, 205126 (2007).

[13] A. K. Soper and M. A. Ricci, Phys. Rev. Lett. 84, 2881 (2000).

[14] L. Bosio, S.-H. Chen and J. Teixeira Phys. Rev. A 27, 1468 (1983).

[15] M. A. Ricci, F. Bruni, and A. Giuliani "Similarities between confined and supercooled water" Faraday Discussion, in press.

[16] C. A. Angell, Science 319, 582 (2008). 
Pressure Effects in Supercooled Water: Comparison between a 2D Model of Water and Experiments for Surf

[17] K. Stokely, M. G. Mazza, H. E. Stanley, and G. Franzese, "Effect of hydrogen bond cooperativity on the behavior of water" e-preprint arXiv:0805.3468 (2008).

[18] R. J. Speedy, J. Phys. Chem. 86, 3002 (1982).

[19] P. G. Debenedetti and M. C. Dantonio, AIChE Journal 34, 447 (1988).

[20] S. Sastry, P. G. Debenedetti, F. Sciortino, and H. E. Stanley, Phys. Rev. E 53, 6144 (1996); L. P. N. Rebelo, P. G. Debenedetti, and S. Sastry, J. Chem. Phys. 109, 626 (1998); Phys. Rev. E 59, 6348 (1999).

[21] H. E. Stanley, Journal of Physics A 12, L329 (1979); H. E. Stanley and J. Teixeira, J. Chem. Phys. 73, 3404 (1980); H. E. Stanley, J. Teixeira, A.Geiger, and R.L.Blumberg, Physica A 106, 260 (1981); A. Geiger and H. E. Stanley, Physical Review Letters 49, 1749 (1982).

[22] E. Schwegler, G. Galli, and F. Gygi, Phys. Rev. Lett. 84, 2429 (2000).

[23] S.-H. Chen, L. Liu, E. Fratini, P. Baglioni, A. Faraone, and E. Mamontov Proc. Natl. Acad. Sci. USA 103, 9012 (2006).

[24] E. Mamontov J. Chem. Phys. 123, 171101 (2005).

[25] H. Jansson, W. S. Howells, and J. Swenson, J. Phys. Chem. B 110, 13786 (2006).

[26] F. Mallamace, M. Broccio, C. Corsaro, A. Faraone, U. Wanderlingh, L. Liu, C. Y. Mou, and S. H. Chen, J. Chem. Phys. 124, 161102 (2006).

[27] J. Swenson, H. Jansson, and R. Bergman Phys. Rev. Lett. 96, 247802 (2006).

[28] V. Lubchenko, P. G. Wolynes, and H. Frauenfelder J. Phys. Chem. B 109, 7488 (2005); P. W. Fenimore, H. Frauenfelder, B. H. McMahon, and R. D. Young Physica A 351, 1 (2005)

[29] J. Swenson, Phys. Rev. Lett. 97, 189801 (2006); S. Cerveny, J. Colmenero, and A. Alegría, Phys. Rev. Lett. 97, 189802 (2006).

[30] S.-H. Chen, L. Liu, and A. Faraone, Phys. Rev. Lett. 97, 189803 (2006).

[31] A. Oleinikova, N. Smolin, and I. Brovchenko, J. Phys. Chem. B 110, 19619 (2006); I. Brovchenko, A. Krukau, and A. Oleinikova Phys. Rev. Lett. 97, 137801 (2006).

[32] S.-H. Chen, et al., J. Chem. Phys. 125, 171103 (2006).

[33] X. Chu, E. Fratini, P. Baglioni, A. Faraone, and S.-H. Chen, Phys. Rev. E 77, 011908 (2008).

[34] P. Kumar, Z. Yan, L. Xu, M. G. Mazza, S. V. Buldyrev, S.-H. Chen. S. Sastry, and H. E. Stanley, Phys. Rev. Lett. 97, 177802 (2006).

[35] J. R. Errington and P. D. Debenedetti, Nature 409, 318 (2001).

[36] A. Faraone, L. Liu, C. Y. Mou, C. W. Yen, and S. H. Chen, J. Chem. Phys. 121, 10843 (2004).

[37] L. Liu, S. H. Chen, A. Faraone, C. W. Yen, and C. Y. Mou, Phys. Rev. Lett. 95, 117802 (2005).

[38] L. Xu, P. Kumar, S. V. Buldyrev, S.-H. Chen, P. H. Poole, F. Sciortino, and H. E. Stanley, Proc. Natl. Acad. Sci. USA 102, 16558 (2005); L. Xu, I. Ehrenberg, S. V. Buldyrev, and H. E. Stanley, J. Phys. C Condensed Matter 18, S2239 (2006); L. Xu, S. V. Buldyrev, C. A. Angell, and H. E. Stanley, Phys. Rev. E 74031108 (2006); L. Xu, S. V. Buldyrev, N. Giovambattista, C. A. Angell, and H. E. Stanley, J. Chem. Phys. (submitted).

[39] G. D’Arrigo, G. Maisano, F. Mallamace, P. Migliardo, and F. Wanderlingh, J. Chem. Phys. 75, 4264 (1981).

[40] C. A. Angell and V. Rodgers, J. Chem. Phys. 80, 6245 (1984).

[41] P. Raiteri, A. Laio, and M. Parrinello, Phys. Rev. Lett. 93, 087801 (2004).

[42] P. Kumar, G. Franzese and H. E. Stanley, Phys. Rev. Lett. 100, 105701 (2008).

[43] P. Kumar, G. Franzese, and H. E. Stanley, J. Phys.: Cond. Matt. 20, 244114 (2008).

[44] P. Kumar, S. V. Buldyrev, F. W. Starr, N. Giovambattista and H. E. Stanley, Phys. Rev. E 72, 051503 (2005).

[45] P. Kumar, F. W. Starr, S. V. Buldyrev, and H. E. Stanley, Phys. Rev. E 75, 011202 (2007).

[46] M. Girardi, M. Szortyka, and M. C. Barbosa, Physica A 386, 692(2007).

[47] F. de los Santos and G. Franzese, in preparation.

[48] S. Han, P. Kumar, and H. E. Stanley, Phys. Rev. E 77, 030201 (2008).

[49] C. A. Angell, S. Borick, and M. Grabow, J. Non-Cryst. Solids 207, 463 (1996).

[50] P. H. Poole, M. Hemmati, and C. A. Angell, Phys. Rev. Lett. 79, 2281 (1997). 
Pressure Effects in Supercooled Water: Comparison between a 2D Model of Water and Experiments for Surf

[51] X.-Q. Chu, A. Faraone, C. Kim, E. Fratini, P. Baglioni, J. B. Leao, S.-H. Chen, "Pressure Dependence of the Dynamic Crossover Temperatures in Protein and its Hydration Water" epreprint arXiv:0810.1228 (2008).

[52] A. Khan, J. Phys. Chem. 104, 11268(2000).

[53] M. Henry, Chem. Phys. Chem. 3, 561(2002).

[54] D. Laage and J. T. Hynes, Science 311, 832 (2006).

[55] R. Casalini, M. Paluch, and C. M. Roland, J. Chem. Phys. 118, 5701 (2003); R. Casalini and C. M. Roland, Phys. Rev. B 71, 014210 (2005). 